\title{
Cavitation in rubber: an elastic instability or a fracture phenomenon?
}

Lefevre, Victor, vlefevre@illinois.edu, Lopez-Pamies, Oscar, University of Illinois at Urbana-Champaign, United States; Ravi-Chandar, Krishnaswamy, University of Texas at Austin, United States

\begin{abstract}
It is by now well established that loading conditions with sufficiently large triaxialities can induce the sudden appearance of internal cavities within elastomeric (and other soft) solids. The occurrence of such a phenomenon, commonly referred to as cavitation, can be attributed to the growth of pre-existing defects into finite sizes. In the first part of this discussion, I will present a new theory within the context of nonlinear elasticity to study the phenomenon of cavitation in rubber that contrary to earlier approaches: (i) allows to consider general 3D loading conditions with arbitrary triaxiality; (ii) applies to general classes of nonlinear elastic solids; and (iii) incorporates direct information on the initial shape, spatial distribution, and mechanical properties of the underlying defects at which cavitation can initiate. The basic idea is to first cast cavitation in elastomeric solids as the homogenization problem of nonlinear elastic materials containing random distributions of zero-volume cavities, or defects. Then, by means of a novel iterated homogenization procedure, exact solutions are constructed for such a problem. These include solutions for the change in size of the underlying cavities as a function of the applied loading conditions, from which the onset of cavitation - corresponding to the event when the initially infinitesimal cavities suddenly grow into finite sizes - can be readily determined. In the second part of the discussion, I will confront the theory with a variety of cavitation experiments with the objective of establishing whether the phenomenon of cavitation is an elastic instability (and hence depends only on the elastic properties of the rubber), or, on the other hand, a fracture process (and hence depends on the fracture properties of the rubber).
\end{abstract}

\section{REFERENCES}

[1] Lefèvre, V., Ravi-Chandar, K., Lopez-Pamies, O. Cavitation in rubber: An elastic instability or a fracture phenomenon? International Journal of Fracture. 2014. Submitted.

[2] Lopez-Pamies, O., Nakamura T., Idiart, M.I. Cavitation in elastomeric solids: I - A defect growth theory. Journal of the Mechanics and Physics of Solids. 2011, 59, 1464-1487.

[3] Lopez-Pamies, O., Nakamura T., Idiart, M.I. Cavitation in elastomeric solids: II - Onset-of-cavitation surfaces for Neo-Hookean materials. Journal of the Mechanics and Physics of Solids. 2011, 59, 1488-1505. 